\title{
British Journal of Visual Impairment
}

September, 2007

\section{Comparison between audio and tactile systems for delivering simple navigational information to visually impaired pedestrians}

\section{Dr. Olinkha Gustafson-Pearce, Professor Eric Billett, Dr. Franjo Cecelja}

Keywords: Tactile interface, tugs, audio interface, navigation, street noise.

\begin{abstract}
Many of the current GPS (Global Positioning Systems) navigation aids use an audio method to deliver navigation information to the user. For the visually impaired person this method can be problematic. The visually impaired pedestrian relies heavily on information contained within the ambient sound environment; for location and orientation information, navigation information, and importantly, safety information. In this paper we present the design of an innovative tactile interface and verification of results obtained through experimental trials. This pilot study compared the efficiency of the tactile interface, to an audio method of delivering simple navigational information. The findings indicate that the tactile interface could be used successfully by blind and sighted pedestrians and may offer advantages over auditory interfaces.
\end{abstract}

\section{Abstract word count: 95}

Article word count: 2706

\section{Introduction}

A number of GPS (Global Positioning Systems) navigation aids have recently been developed and marketed, both for general use, (in-car and personal systems) and for use by specific user groups. Amongst these 'specific use' systems is the equipment 
developed for use by visually impaired pedestrians (TalkNav, 2007; Trekker, 2007; The Voice, 2007). The majority of these GPS based systems use an audio feedback to impart navigation information to the user. In the case of the visually impaired pedestrian, this form of information transfer is problematic (Shoval, Ulrich, 2003). The visually impaired pedestrian relies heavily on information contained within the ambient sound environment; for location and orientation information, navigation information, and importantly, safety information. The visually impaired pedestrian needs to be able to hear ambient sounds, such as the approach of a vehicle or other pedestrians. The common use of the 'ear-bud' speakers, mutes or degrades the ability to hear these important sounds. Even with a speaker mounted in another position, for example, shoulder mounted speakers, the user still finds problems with simultaneous processing of diverse sound signals. This can lead to 'information overload' of the audio channels and cause the user significant problems (Tugs research, 2007). To address these problems, a tactile interface has been designed and developed, and has been called the tugs (tactile user guidance system) interface (The tugs system, 2007). Many of the aids available for visually impaired pedestrians utilise the haptic perception, (the cane, Braille, tactile maps) therefore tactile interfaces are an effective method of information gathering. The majority of the aids currently in use, employ the active tactile method, which requires the user to engage in information gathering/processing in order to disseminate the information. Tugs uses a passive tactile feedback, which does not require the user to engage in information gathering/processing; the messages are given and the user responds. This method will be further discussed in section 2.3.

In this paper we present the verification results obtained through experimental trials, which were designed to compare results from navigation protocols delivered through the audio channel, to the same protocols delivered by the tugs system.

\subsection{Design of the tugs system}

A test system was designed which allowed easy movement of the actuators (see figure 1). 


\section{[FIGURE 1 ABOUT HERE]}

The system took the form of a harness, which is worn under the outer layer of clothing but over lightweight underwear. The actuators are small and lightweight and can be incorporated into a form of apparel that is appropriate to the user. For the test phase the actuators are activated from a control panel. The buttons on this panel relate to specific actuators on the harness. In the trials the control panel is held and activated by an operator. The potential also exists to automate this activation using remotely held maps and pre-figured route plans.

\subsection{Base and high order message structures}

An analysis was made of the range of messages that the blind or visually impaired pedestrian requires, in order to safely navigate through a complex environment. It was seen that all messages could be divided into two categories. These were the 'command' messages, (turn left/right, go up/down and stop) which could be considered as the purely navigational requirements and the 'conversational' messages, which would be best suited to an intelligent interaction with a guide. These conversational interactions were already possible with the existing methods of information transference through the GPS systems (using the audio channel) but, as previously stated, the user found difficulties if these channels were in constant operation. Therefore if the 'command' messages are removed from the audio channel this reduces the amount of information transferred through the audio channel.

The navigational protocols (message modes) have been separated into a base and high order structure. Base instructions are made up of the simple or low order information contained within the navigational protocols. These comprise of directional instructions, 'stop' warning information and may also be set for transmission of other information of a 'single message' nature. The high order information can still be transferred through the audio channel and consists of information that is more appropriate for natural language. This conversational interaction might relate to correct item identification, bus or train route displays, shopping, signage etc.. 


\subsection{The Natural Reference Frame}

In her extensive studies with blind subjects, Susanna Millar (Millar, 1994) has observed that the body midline seems to be 'used systematically as a reference frame, so that locations and directions are to be specified in relation to it.' In initial trials with the tugs system, in which the author approached more than 20 adults at random, the actuators were moved around the harness and the subjects were asked to move in a direction that 'felt right'. It was found that all subjects moved towards the signal in the left/right (actuators 3 and 5) scenario and indicated up/down (actuators 1 and 2) when these actuators were activated. It was further found that the positions shown in figure 1 were the most effective in separating the signal and giving clear messages to the user. In further trials, including the one reported on in this paper, the same pattern was to be found. Therefore, a navigational interface, which enhanced this natural reference frame, theoretically would allow the user to respond in an extemporaneous manner to navigational information. The tugs interface uses passive tactile feedback to the user, thereby utilising the areas that use instinctive rather than analytical mental processing. In this way it would reduce the potential for 'information overload' that are observed with the current (audio) navigational interfaces.

\subsection{Method}

The purpose of this trial is to establish the efficiency of the tugs system by direct comparison between tugs, and the commonly used voice interfaces. It was determined that the trial environment should place the subjects and the interface under pressure, but still maintain high levels of safety for the participants. Therefore the main parameters for this trial were designed in order to test the tugs interface in controlled conditions to ascertain its performance in comparison to an audio method of delivering navigation information.

This trial is in two sections; by direct comparison and by testing it with street noise. Further information about the tugs interface was elicited in the response sheets, with the participants answering staged questions regarding the comfort and placing of the actuators. It was felt that some users might find the position of the actuators, or level of the vibrations uncomfortable. The participants were also asked whether they were 
confused by the tugs signal. The trial was designed and then tested with three volunteers. Responses were noted and specific factors were changed (street noise instead of white noise, see section 3.5.) before the trial commenced.

\subsection{Participants}

The participants in the trial were placed into two groups defined by visual ability.

\section{2a. Visually Impaired Volunteers}

The visually impaired group consisted of seven volunteers, details shown in chart 1 .

\section{[TABLE 1 ABOUT HERE]}

\section{2b. Sighted Volunteers}

The sighted group consisted of fourteen volunteers, details shown in chart 2.

\section{[TABLE 2 ABOUT HERE]}

Regarding language, 7 listed English as their first language, 3 were bilingual with English as one of their languages, 4 were not British and had average English language skills. Of these, all 4 were attending an undergraduate course at a British university as exchange students.

\subsection{Location of Trial}

The trial took place in audio and engineering laboratories at Brunel University. All extraneous sound and movement were eliminated from the areas during the trial. The area was set up as illustrated in figure 2 .

\section{[FIGURE 2 ABOUT HERE]}

The trial subject stood facing towards the speakers. The area was marked on the floor to ensure that each subject assumed the same position during the trial. The tugs operator held the control panel, which had the cable leading to the tactile actuators. 
The assistant operator controlled the audio and noted the responses of the subject on the response sheets. The duration of each trial averaged at 20 minutes for both groups.

\subsection{Tugs Interface}

The subjects were introduced to the tugs interface and were encouraged to experience the level of vibrotactile stimuli with their hands before the equipment was placed on them. The full harness was employed but only actuators 3 and 5 were used in this series of tests. See figure 1 . The tugs interface was placed on the subject, over the top of a lightweight undergarment such as a vest or t-shirt. The tugs operator stood behind the subject and activated the vibrotactile stimuli through the tugs control panel.

\section{5a. Street Noise background sound recording and audio files}

The street noise program was constructed from generic, audio library, background files. Three separate files were used. These were New York, Rome and London. The files were mixed and included sirens, revving engines and shouting. The end file was intended to be varied with the sirens and other noises being unexpected and 'startling' to the listener. This was to avoid the subject becoming habituated to the audio and therefore being able to block out or ignore the sounds. In the trial design development stage, white noise and grey noise were experimented with, but it was found that the subjects very quickly became habituated to these sounds. This final, street noise, audio file had constant noise, with a 10dB (decibel) difference in sound levels. It was 10 minutes long and looped. The street noise sound file was played to the subject through the two speakers mounted as shown in figure 2. To determine the $\mathrm{dB}$ level for sets $1 / 3 \mathrm{a}-1 / 4 \mathrm{~b}$ the documents WHO document Guidelines for Community Noise and The National Noise Incidence Study 2000/2001 were referred to. Based on these documents, levels for the trials were as follows; sets $1 / 3 \mathrm{a}, 1 / 3 \mathrm{~b}-70 \mathrm{~dB}$ and sets $1 / 4 \mathrm{a}$, $1 / 4 \mathrm{~b}-90 \mathrm{~dB}$. The $\mathrm{dB}$ monitor was placed in the subject position and levels were monitored at the beginning of each session.

\section{5b. Audio sound files}


The spoken left/right instruction audio file was recorded for the trials and used a female voice. A 1 second interval between signals was used and the audio was delivered to the participant through a single ear-piece.

\subsection{Tugs signal}

The vibrotactile signals had a binomial distribution, in that either the left or the right actuator was activated. The tugs signals were equivalent in time duration and event frequency to the audio instructions.

\section{Results}

Noise settings: sets 1/1, 1/2 - no background noise. Street noise present in: sets 1/3a, 1/3b - 70dB and sets 1/4a, 1/4b - 90dB. Across all sets, each participant was given 50 tugs signals and 50 audio signals.

\subsection{Comparisons between tugs and audio signals}

The scores from the visually impaired participants show two errors across all sets. Both these errors are in the audio sections of sets $1 / 3 b$ and $1 / 4 b$. There were no errors on the tugs signals.

The scores from the sighted participants show 3 errors in the tugs sections and 22 errors in the audio section with the majority of the errors (13) made in set $1 / 3 b$ by two of the subjects. Both these subjects listed English as their first language and in the further comment section, stated that they had problems with defining left/right under normal circumstances. They commented that once they had made an error it was difficult to resume accurate responses. When the scores from all groups are compiled table 1 is obtained.

\section{[TABLE 3 ABOUT HERE]}

Over all sets and to all participants, 1050 audio signals and 1050 tugs signals were given. Of this, tugs scored an error in 3 instances and the audio scored an error of 24. This shows a rating of tugs .997 correct and audio .977 correct. In both cases the error 
levels are low and, on the assumption that the failures take the form of a Poisson distribution, this statistical analysis was performed.

The details of the analysis are shown in Notes.

Using this analysis the $\mathrm{Z}$ variable evaluates to, $\mathrm{Z}=130$.

The value of $\mathrm{Z}$ is $>>1.96$, therefore we can safely reject the nul hypothesis at the .05 level and conclude that the two samples are not the same mean, and that tugs performs better than audio in this situation.

\subsection{Response sheets}

Response sheets were designed for use in this trial and responses from each set were recorded. In addition the subjects were asked to rate their comfort levels for the tugs interface on a nominal score rating, from very uncomfortable (1) to very comfortable (5). They were asked to rate, [a] strength of vibration and [b] placing of the actuator. There was also a space in these sections for the subject to make any comments about the test. During the trial the trial assistant completed the sheets as the subject responded to the various components.

\section{2a. Results for signal strength and placing of the actuators}

\section{[TABLE 4 ABOUT HERE]}

The averaged scores are shown in table 4. It can be seen from this data that the majority of participants in both groups scored high levels of comfort in the positioning of the actuators and signal strength. In this trial the participants were not given a range of signal strengths and positions from which to choose a preference, so they did not have a comparison range. The ability of the user to vary the strength of the signal and the position of the actuators should be investigated in further trials. However this pilot study shows that, overall, users found the interface comfortable, and did not experience significant levels of discomfort.

\section{2b. Subjects comments noted during the trials}

Many of the subjects recorded similar comments during the trials, with most comments related to the tugs interface. A significant number of the participants noted 
that the tugs interface felt 'strange, not uncomfortable, just unusual'. Many of the subjects noted that tugs felt 'intuitive' and that with a longer period of use they would become 'very comfortable' with using this interface.

\section{Conclusion}

This trial was to determine whether the tugs and the audio signals would each be effective at delivering simple navigational instructions to a user and whether one system was more effective than the other. The tugs signal was delivered to the subject through the vibrotactile interface utilising the natural reference frame, and the audio instructions were delivered through a single ear-piece. The increasing level of street noise through sets $1 / 3 a-1 / 4 b$ was not a factor in the error levels, with the majority of the audio errors made whilst street noise was at $70 \mathrm{~dB}$. In a 'natural' pedestrian environment however, the use of an ear-bud speaker or headset, would compromise the pedestrians' ability to attend to ambient sounds. The low level of errors in the tugs responses (1050 signals, 3 errors with group scores combined) show it to be effective at delivering simple navigational information. It appears not to be affected by ambient noise and the method of delivering the messages (vibrotactile stimulation) may have more immediacy and be more 'intuitive' than the same message delivered through the medium of language. Tugs was particularly effective at delivering navigational information under these trial conditions to visually impaired pedestrians, (350 signals, 0 errors) for whom the ability to attend to ambient sounds in the environment is vital.

\section{Notes}

The Poisson Distribution 


$$
\begin{aligned}
& Z=\left(\left(\bar{\chi}_{1}-\bar{\chi}_{2}\right)-\left(\mu_{1}-\mu_{2}\right)\right) \div \sqrt{\frac{\sigma_{1}^{2}}{n_{1}}+\frac{\sigma_{1}^{2}}{n_{2}}} \\
& Z=24-3 \div \sqrt{\frac{3}{1050}+\frac{24}{1050}} \\
& Z=21 \div \sqrt{\frac{27}{1050}} \\
& Z=21 \div \sqrt{.0257} \\
& Z=21 \div .1603 \\
& Z=130
\end{aligned}
$$

\section{References}

Humanware (2007, 15 March). Products: GPS Orientation Solution: Trekker. [http://www.humanware.com]

Millar, S., 1994. Understanding and Representing Space. Oxford University Press. Oxford. pp.144.

Shoval S., Ulrich I., 2003. NavBelt and Sonic Guide. Robotics-Based ObstacleAvoidance Systems for the Blind and Visually Impaired. Borenstein J. Invited article for the IEEE Robotics and Automation Magazine, Special Issue on Robotics in BioEngineering. 10(1), pp. 9-20.

TalkNav (2007, 15 March). Product Details. [http://www.talknav.co.uk]

The National Noise Incidence Study 2000/2001 (United Kingdom): (2001) Volume 1 - Noise Levels.

The tugs system. (2007, 15 March). http://www.dea.brunel.ac.uk/tugs

The Voice (2007, 15 March). The Voice [http://www.seeingwithsound.com/voice.htm

Tugs research (2007, 15 March). A GPS system for use by visually impaired pedestrians has been developed at Brunel University. The problems outlined, in reference to masking of audio messages, were identified in trials with the system. For further information on the research at Brunel University please refer to:

http://www.dea.brunel.ac.uk/tugs/research

World Health Organisation (WHO). (2004). Guidelines for Community Noise. In the European Union countries about $40 \%$ of the population are exposed to road traffic noise with an equivalent sound pressure level exceeding $55 \mathrm{~dB}(\mathrm{~A})$ daytime and $20 \%$ are exposed to levels exceeding $65 \mathrm{~dB}(\mathrm{~A})$ Data collected alongside densely travelled 
roads were found to have equivalent sound pressure levels for 24 hours of 75 to 80 $\mathrm{dB}(\mathrm{A})$.

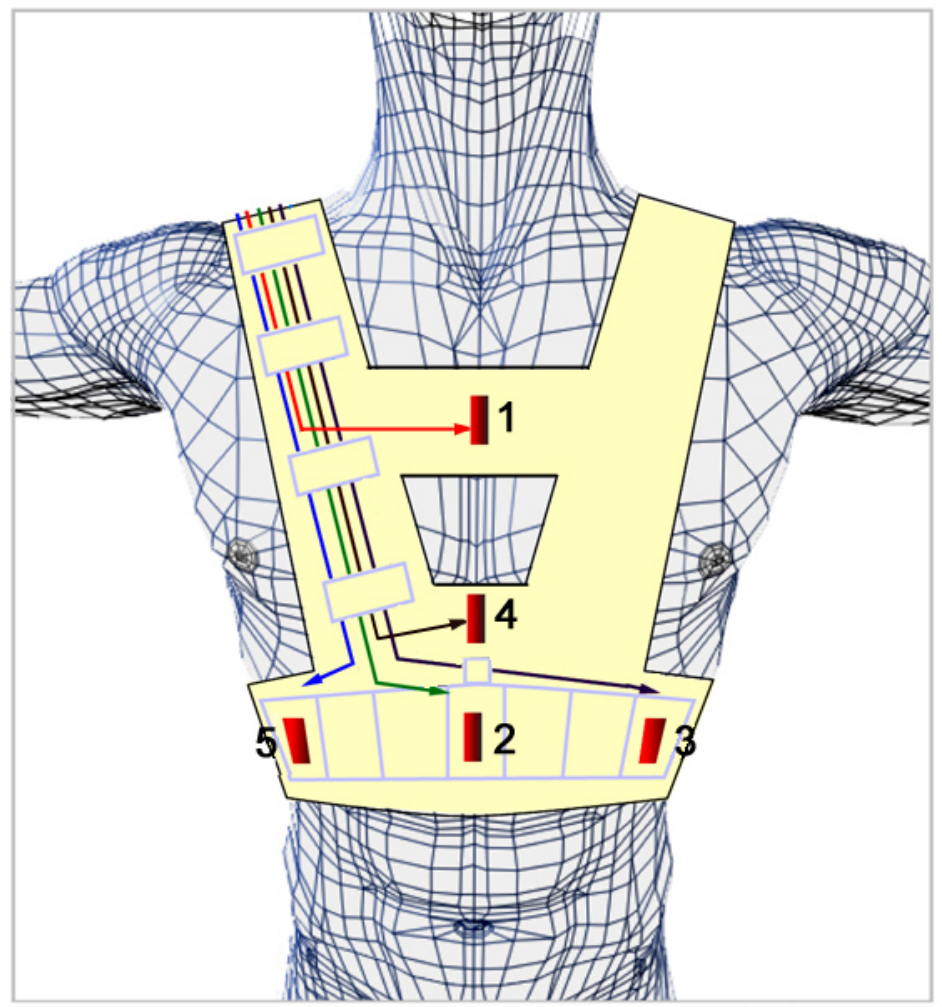

figure 1. Tugs and harness 


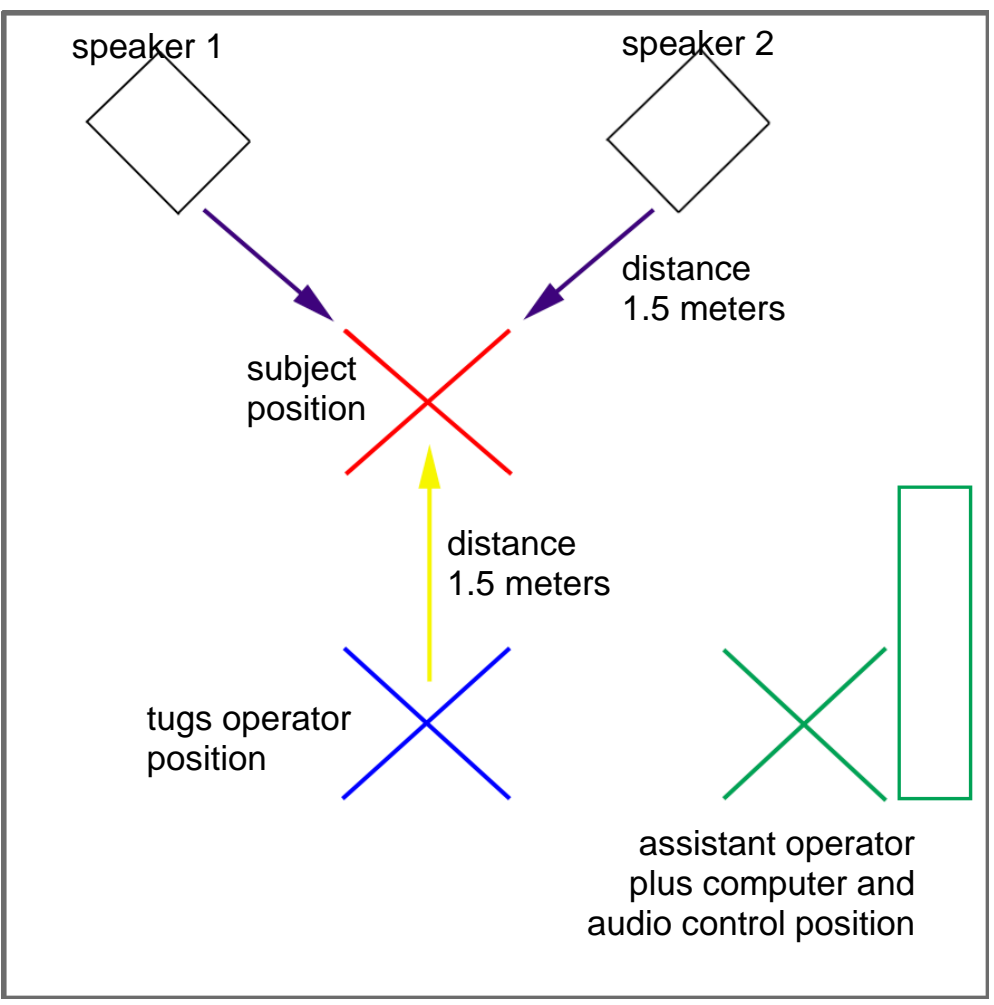

figure 2. Equipment placing

\begin{tabular}{|c|c|c|c|c|c|}
\hline Subject number & Vision & Aid & Language & Age & Gender \\
\hline 001 & Blind & Guide dog & English & $25-35$ & Male \\
\hline 002 & Blind & Guide dog & English & $25-35$ & Female \\
\hline 003 & Blind & Guide dog & English & $30-40$ & Female \\
\hline 004 & Blind & Guide dog & English & $30-40$ & Female \\
\hline 005 & Low vision & Long cane & English & $45-60$ & Male \\
\hline 006 & Low vision & Long cane & English & $45-60$ & Male \\
\hline 007 & Low vision & Long cane & English & $45-60$ & Male \\
\hline
\end{tabular}

table 1. Visually Impaired subjects

\begin{tabular}{|c|c|c|c|c|}
\hline Subject number & Visual ability & Occupation & Age & Gender \\
\hline $101-108$ & Good & Student & $20-30$ & Male/Female \\
\hline 110 & Contact lenses & Student & $20-30$ & Male \\
\hline $111-113$ & Spectacles & Student & $20-30$ & Male/Female \\
\hline 114 & Spectacles & Photographer & $45-60$ & Female \\
\hline
\end{tabular}

table 2. Sighted subjects 


\begin{tabular}{|l|c|c|c|c|}
\hline & TUGS & & AUDIO & \\
\hline & correct & incorrect & correct & incorrect \\
\hline $\begin{array}{l}\text { set s 1/1a and 1/2a } \\
\text { no street noise }\end{array}$ & 209 & 1 & 208 & 2 \\
\hline $\begin{array}{l}\text { sets 1/3a and 1/3b } \\
\text { street noise at 70dB }\end{array}$ & 419 & 1 & 403 & 17 \\
\hline $\begin{array}{l}\text { sets 1/4a and 1/4b } \\
\text { street noise at 90dB }\end{array}$ & 419 & 1 & 415 & 5 \\
\hline
\end{tabular}

table 3. Both groups combined

\begin{tabular}{|l|l|c|}
\hline \multirow{2}{*}{} & \multicolumn{2}{|c|}{ Score average } \\
\cline { 2 - 3 } & VIP & Sighted \\
\hline is the strength/level of the vibration comfortable? & 4.3 & 4.3 \\
\hline Is the placing of the actuator/vibrator comfortable? & 4.3 & 4.4 \\
\hline
\end{tabular}

table 4. Questionnaire responses. ( $\mathrm{VI}=$ visually impaired participants. $5=$ very comfortable, 1 = very uncomfortable) 